\title{
Primary aldosteronism-associated cardiomyopathy: Clinical-pathologic impact of aldosterone normalization is
}

\author{
Andrea Frustaci ${ }^{\mathrm{a}, \mathrm{d}, *}$, Claudio Letizia ${ }^{\mathrm{b}}$, Romina Verardo ${ }^{\mathrm{d}}$, Claudia Grande ${ }^{\mathrm{d}}$, Marco Francone ${ }^{\mathrm{c}}$, Luigi Sansone ${ }^{\mathrm{e}}$, \\ Matteo Antonio Russo e,f , Cristina Chimenti a,d \\ a Department of Cardiovascular, Respiratory, Nephrologic, Anesthesiologic and Geriatric Sciences, La Sapienza University, Italy \\ b Department of Translational and Precision Medicine, Center for Secondary Hypertension, La Sapienza University, Italy \\ c Department of Radiological, Oncological and Pathological Sciences, La Sapienza University, Rome, Italy \\ d Cellular and Molecular Cardiology Lab, IRCCS L. Spallanzani, Rome, Italy \\ e Cellular and Molecular Pathology Lab, IRCCS S. Raffaele Pisana, Rome, Italy \\ ${ }^{\mathrm{f}}$ MEBIC Consortium, San Raffaele Open University, Rome, Italy
}

\section{A R T I C L E I N F O}

\section{Article history:}

Received 27 March 2019

Received in revised form 3 June 2019

Accepted 19 June 2019

Available online 20 June 2019

\section{Keywords:}

Cardiomyopathy

Water overloading

Myocardial aldosterone

\begin{abstract}
A B S T R A C T
Background: Primary aldosteronism (PA) causes a cardiomyopathy (CM) which substrate and evolution after aldosterone normalization are unreported.

Methods: Four male patients with aldosterone-secreting adrenal adenoma and cardiomyopathy (PACM, group A) were evaluated with 2D-echo, Magnetic Resonance (CMR), coronary angiography and left ventricular endomyocardial biopsy. Biopsy samples were processed for histology, electron microscopy, immunohistochemistry, and Western Blot analysis of myocardial aldosterone receptors and aquaporin 1 and 4.

Results were compared with endomyocardial samples from 5 patients with hypertensive cardiomyopathy of equivalent severity and normal plasma aldosterone (group B) and surgical samples from 5 controls (group C). One PACM patient was re-examined with CMR and endomyocardial biopsy 12 months after adrenalectomy with aldosterone and cardiac normalization.

Results: Coronary arteries were normal in all. Group A showed prominent myocardial hypertrophy and fibrosis, with water accumulation in the cytosol and organelles of cardiomyocytes and microvascular smooth muscle cells, associated to reduced myofibril concentration and 2.8-fold increase in myocardial aldosterone receptors and aquaporin 1. At CMR, LGE areas were diffusely present. After aldosterone normalization, cardiomyocyte diameter reduced with disappearance of intracellular vacuoles, recovery of electron-density of cytosol and cell organelles, and myofibrillar content, persisting fibrosis and down-regulation of aldosterone receptors and aquaporin 1 channels. At CMR, myocardial mass reduced with recovery of cardiac contractility. LGE signal remained unchanged.

Conclusion: PACM is a reversible entity characterized by over-expression of aldosterone receptors and aquaporin 1 . It induces a reversible intracellular water overloading causing impaired cardiomyocyte relaxation, contraction and ultrastructural integrity.
\end{abstract}

(c) 2019 Elsevier B.V. All rights reserved.

\section{Introduction}

Aldosterone hypersecretion has been shown to adversely affect human cardiovascular system through the homologous receptor localized in the heart and vessels [1].

Specifically, hyper-stimulation of cardiovascular aldosterone receptors has been associated with hypokalemic hypertension, myocardial

\footnotetext{
is "The authors take responsibility for all aspects of the reliability and freedom from bias of the data presented and their discussed interpretation."

* Corresponding author at: Department of Cardiovascular, Respiratory, Nephrologic, Anesthesiologic and Geriatric Sciences, La Sapienza University, Viale del Policlinico 155, 00161 Rome, Italy.

E-mail address: biocard@inmi.it (A. Frustaci).
}

hypertrophy with sarcomere gene overexpression, myocardial fibrosis, left ventricular diastolic and systolic dysfunction [2,3]. Interestingly, cardiac hypertrophy and diastolic dysfunction manifest earlier than arterial hypertension suggesting a direct cardiac hormonal action [4]. In clinical practice, recognition of a hyperaldosteronism has therapeutic implications as the administration of aldosterone inhibitors may improve patients' outcome.

To this regard, a convincing evidence comes from Rales [5], Emphasis-HF [6,7] and Ephesus trial [8] where inhibition of myocardial aldosterone receptors has been followed by reduction of left ventricular hypertrophy and decrease in mortality and cardiovascular-related hospitalizations compared with placebo. As a consequence, aldosterone inhibitors are recommended by the current guidelines in the pharmacologic treatment of heart failure $[9,10]$. 
Nevertheless, pathologic changes induced by abnormal aldosterone secretion in human myocardium are unavailable, while the molecular mechanisms involved are so far poorly understood.

In the present study, histological and ultrastructural myocardial changes observed in 4 patients with primary aldosteronism-associated cardiomyopathy are reported. In one subject a control biopsy has been obtained 12 months after surgical removal of an aldosterone-secreting adrenal adenoma with aldosterone normalization and recovery of cardiac function. Morphological analysis of endomyocardial samples has been implemented with assessment of myocardial aldosterone receptors and aquaporin 1 and 4 channels.

\section{Methods}

\subsection{Patient population}

Four male patients aged 32,36, 56 and 69 years respectively (group A) were admitted because of dyspnea on effort (NYHA class 2/3) and a cardiomyopathy (left ventricular (LV) ejection fraction (EF) 40,30, 25 and 38\% respectively) associated to systemic arterial hypertension secondary to aldosterone secreting adrenal adenoma. Adenoma was located by nuclear magnetic resonance in the right adrenal gland in patient 1 and 3 while was detected in the left one in the patient 2 and 4 . Diagnosis of primary aldosteronism was realized in the 4 affected patients for the presence of plasma aldosterone concentration $\gg 15 \mathrm{ng} / \mathrm{dL}$ and an upright plasma aldosterone concentration/plasma renin activity ratio $\gg 30$ (ARR). Furthermore, our patients underwent a saline infusion $(0.9 \% \mathrm{NaCl}$ at $500 \mathrm{ml} / \mathrm{h})$ that failed to decrease plasma aldosterone concentration to $\ll 7 \mathrm{ng} / \mathrm{dL}$. For comparison 5 patients ( $3 \mathrm{M}$, mean age $53.7 \pm 12.5 \mathrm{ys}$ ) with essential arterial hypertension and reduced LV function (group B) and 5 controls (group C, 4M, mean age $55 \pm 11.3$ ys) were studied. For morphological studies, controls were represented by papillary muscle fragments coming from patients with mitral stenosis and normal left ventricular dimension and function undergoing mitral valve replacement. (Table 1) The 69-year old patient underwent surgical removal of adrenal adenoma. It followed a normalization of plasma aldosterone levels ( $6 \mathrm{ng} / \mathrm{dL}$ ) that was paralleled by decrease of left ventricular mass and recovery of contractility. A follow-up left ventricular endomyocardial biopsy was obtained after 12 months for histological and molecular evaluation (Table 2 supplementary material).

\subsection{Cardiac studies}

All patients underwent ECG, 2D-echocardiogram, cardiac magnetic resonance and invasive cardiac studies including cardiac catheterization, coronary angiography and left ventricular endomyocardial biopsy, after written informed consent and approval by local ethical committee, to clarify the cause and mechanism of cardiomyopathy.

\subsubsection{Cardiac magnetic resonance}

In all patients a standard cardiac magnetic resonance (CMR) examination was performed using a 1.5-T system (Magnetom Avanto; Siemens Medical Systems, Germany) equipped with multi-element phased array surface coil.

Table 1

Comparison of clinical and endomyocardial biopsy data among human primary aldosteronism (Group A), hypertensive cardiomyopathy (Group B), and normal controls (Group C).

\begin{tabular}{|c|c|c|c|c|}
\hline & $\begin{array}{l}\text { Group A } \\
\mathrm{n}=4\end{array}$ & $\begin{array}{l}\text { Group B } \\
\mathrm{n}=5\end{array}$ & $\begin{array}{l}\text { Group C } \\
\mathrm{n}=5\end{array}$ & $p$ value \\
\hline Age & $47.5 \pm 16.7$ & $48.4 \pm 8.8$ & $47.2 \pm 8.8$ & NS \\
\hline Sex & $4 \mathrm{M}$ & $3 \mathrm{M} ; 2 \mathrm{~F}$ & $4 \mathrm{M} ; 1 \mathrm{~F}$ & NS \\
\hline BMI $\left(\mathrm{kg} / \mathrm{m}^{2}\right)$ & $37.25 \pm 1.7$ & $27.7 \pm 4.2$ & $22.16 \pm 3.3$ & $\begin{array}{l}p \ll 0.05 A \text { vs } B, B \text { vs } C \\
p \ll 0.001 A \text { vs } C\end{array}$ \\
\hline $\mathrm{SBP}(\mathrm{mmHg})$ & $165 \pm 12.9$ & $162 \pm 10.8$ & $121 \pm 7.4$ & NS for A vs B \\
\hline $\mathrm{DBP}(\mathrm{mmHg})$ & $102.5 \pm 6.5$ & $96.2 \pm 8$ & $71 \pm 7.4$ & $\begin{array}{l}p \ll 0.05 \text { A vs } C \\
p \ll 0.001 \text { B vs C }\end{array}$ \\
\hline HR (bpm) & $71 \pm 25$ & $68.2 \pm 15.9$ & $78 \pm 10.4$ & NS \\
\hline \multicolumn{5}{|l|}{ Blood values } \\
\hline Creatinine (mg/dl) & $0.81 \pm 0.1$ & $0.9 \pm 0.6$ & $0.9 \pm 0.2$ & NS \\
\hline $\mathrm{Na}(\mathrm{mmEq} / \mathrm{L})$ & $140.25 \pm 2.1$ & $142.6 \pm 27.4$ & $123.6 \pm 50.9$ & NS \\
\hline $\mathrm{K}(\mathrm{mmEq} / \mathrm{L})$ & $3.3 \pm 0.7$ & $4.4 \pm \overline{0.6}$ & $4.1 \pm 1.7$ & NS \\
\hline $\mathrm{PAC}$ (ng/dl) & $20 \pm 1.6$ & $16.3 \pm 8.1$ & $7.9 \pm 3$ & $p \ll 0.001 \mathrm{~A}$ vs $C$ \\
\hline PRA (ng/ml/h) & $0.25 \pm 0.1$ & $3.8 \pm 1.6$ & $1.44 \pm 0.8$ & $p \ll 0.05$ A vs $B, A$ vs $C, B$ vs $C$ \\
\hline PAC/PRA ratio (ng/dl:ng/ml/h) & $103.3 \pm 66.6$ & $5.50 \pm 5.47$ & $6.6 \pm 4$ & $p \ll 0.001 \mathrm{~A}$ vs $\mathrm{B}, \mathrm{A}$ vs $\mathrm{C}$ \\
\hline $\operatorname{AUR}(\mu \mathrm{g} / 24 \mathrm{~h})$ & $33.5 \pm 1.3$ & $27.08 \pm 4.6$ & $11.6 \pm 8.3$ & $p \gg 0.05 \mathrm{~A}$ vs $\mathrm{C}, \mathrm{B}$ vs $\mathrm{C}$ \\
\hline ACTH $(\mathrm{pg} / \mathrm{ml})$ & $12.05 \pm 3.2$ & $15.8 \pm 6.8$ & $13.14 \pm 4.1$ & NS \\
\hline PC $(n m o l(l)$ & $406 \pm 55.2$ & $390 \pm 128.3$ & $519.6 \pm 153.5$ & NS \\
\hline UFC (nmol/24 h) & $149.3 \pm 12.6$ & $178.9 \pm 15.5$ & $92.4 \pm 62.5$ & $p \ll 0.05 \mathrm{~B}$ vs $\mathrm{C}$ \\
\hline Ur Metanephrine $(\mu \mathrm{g} / 24 \mathrm{~h})$ & $31.7 \pm 12.4$ & $28.7 \pm 28.9$ & $29.02 \pm 15.6$ & NS \\
\hline \multicolumn{5}{|l|}{ Echocardiography } \\
\hline $\operatorname{MWT}(\mathrm{mm})^{*}$ & $14.7 \pm 1$ & $12.6 \pm 0.3$ & $9.3 \pm 2$ & $p \ll 0.01 \mathrm{~A}$ vs $\mathrm{B}, \mathrm{B}$ vs $\mathrm{C}, \mathrm{A}$ vs $\mathrm{C}$ \\
\hline LVEDV† & $63.7 \pm 3.5$ & $66 \pm 2.9$ & $46.6 \pm 6.3$ & $p \ll 0.05$ A vs $C, B$ vs $C$ \\
\hline $\mathrm{EF} \% \ddagger$ & $41.25 \pm 7.9$ & $42.2 \pm 5.5$ & $60.8 \pm 5.6$ & $\begin{array}{l}p \ll 0.01 \text { A vs } C \\
p \ll 0.001 \text { B vs C }\end{array}$ \\
\hline \multicolumn{5}{|l|}{ CMR data } \\
\hline Myocardial mass (g) & $225.3 \pm 52.2$ & $162 \pm 21.4$ & $90.6 \pm 16.7$ & $\begin{array}{l}p \ll 0.05 A \text { vs B } \\
p \ll 0.001 A \text { vs C, B vs C }\end{array}$ \\
\hline LV mass index (g/m2) & $108.9 \pm 24.9$ & $90.2 \pm 10$ & $53.6 \pm 5.4$ & $\begin{array}{l}p \ll 0.01 \text { A vs } C \\
p \ll 0.001 \text { B vs } C\end{array}$ \\
\hline \multicolumn{5}{|l|}{ Endomyocardial biopsy studies } \\
\hline Cardiomyocyte diameter $(\mu \mathrm{m})$ & $31 \pm 0.8$ & $24 \pm 1.4$ & $15 \pm 1.6$ & $p \ll 0.001 \mathrm{~A}$ vs $\mathrm{B}, \mathrm{A}$ vs $\mathrm{C}, \mathrm{B}$ vs $\mathrm{C}$ \\
\hline Fibrosis (\%) & $14.9 \pm 3.8$ & $6.26 \pm 1.6$ & $1.44 \pm 0.4$ & $\begin{array}{l}p \ll 0.01 \mathrm{~A} \text { vs } \mathrm{B} \\
p \ll 0.001 \mathrm{~A} \text { vs } \mathrm{C}, \mathrm{B} \text { vs } \mathrm{C}\end{array}$ \\
\hline Aldosterone receptor grade $(0 / 4)$ & $1.0 \pm 0.2$ & $3.3 \pm 0.7$ & $3.54 \pm 0.5$ & $\begin{array}{l}p \ll 0.001 \mathrm{~A} \text { vs } B \\
\mathrm{~A} \text { vs } \mathrm{C}\end{array}$ \\
\hline AQP1 grade $(0 / 4)$ & $3.7 \pm 0.4$ & $1.24 \pm 0.4$ & $1.18 \pm 0.2$ & $p \ll 0.001 \mathrm{~A}$ vs $\mathrm{B}, \mathrm{A}$ vs $\mathrm{C}$ \\
\hline Aldosterone receptor Western blot (arbitrary units) & $26,881 \pm 3247$ & $9766 \pm 561$ & $8878 \pm 562$ & $p \ll 0.001 \mathrm{~A}$ vs $\mathrm{B}, \mathrm{A}$ vs $\mathrm{C}$ \\
\hline AQP1 Western blot (arbitrary units) & $24,243 \pm 6423$ & $8444 \pm 742.4$ & $7934 \pm 768$ & $p \ll 0.001 \mathrm{~A}$ vs $\mathrm{B}, \mathrm{A}$ vs $\mathrm{C}$ \\
\hline \multicolumn{5}{|l|}{ Ultrastructural findings } \\
\hline Myofibrillar area (\% of total cell area \pm SD) & $23 \pm 7.3$ & $53.3 \pm 6.8$ & $47 \pm 2.8$ & $p \ll 0.01 \mathrm{~A}$ vs $\mathrm{B}, \mathrm{A}$ vs $\mathrm{C}$ \\
\hline Mitochondrial area (\% of total cell area \pm SD) & $15.3 \pm 2.5$ & $30 \pm 5.7$ & $30.8 \pm 8.3$ & $p \ll 0.05$ A vs $B, A$ vs $C$ \\
\hline SR cisternae area (\% of total cell area \pm SD) & $16.5 \pm 4.7$ & - & - & \\
\hline
\end{tabular}

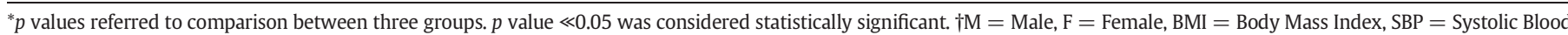

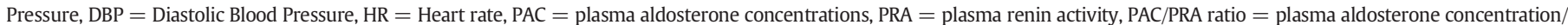

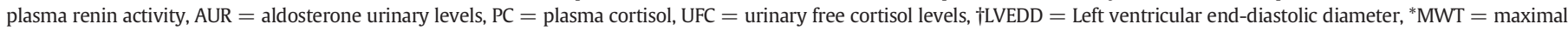
wall thickness, $\mathrm{t} E F=$ ejection fraction.

Bold values indicates significance at $p<0,05 \mathrm{~A}$ vs $\mathrm{C}$, B vs $\mathrm{C}$. 
Scanning protocol consisted with the acquisition of TSE T2w-STIR, cine SSFP (steadystate free precession) and delayed enhancement (DE) sequences 12-18 min after gadolinium administration; on short-axis images left ventricle was completely encompassed from the base to the apex acquiring a total of 10-12 images.

T1 mapping imaging was also performed in all cases before and after contrast using a modified look-locker (MOLLI) sequence acquired in short axis at basal- mid and apica segments, before and after contrast.

\subsubsection{Histology and electron microscopy}

Endomyocardial biopsy samples were processed for routine histological analysis and for transmission electron microscopy (TEM). In addition, two myocardial fragments each patient were processed for assessment of aldosterone receptors and aquaporin channels.

For histological analysis the endomyocardial samples were fixed in $10 \%$ buffered formalin and paraffin embedded. Five micron thick sections were stained with hematoxylin \& eosin, Masson trichrome and Miller's Elastic Van Gieson.

For TEM, samples were fixed in $2 \%$ glutaraldehyde in a $0.1 \mathrm{M}$ phosphate buffer, at $\mathrm{pH} 7.3$, post fixed in osmium tetroxide and processed following a standard schedule for embedding in Epon resin. Ultrathin sections were stained with uranyl acetate replacement stain (EMS \#22405, from EMS, Hatfield, Pa, USA) and lead hydroxide. A Jeol-1400-plus TEM was used for observation and photographic analysis.

Morphometric analysis of subcellular cytoplasmic components (sarcomeres, mitochondria, vacuoles and dilated sarcoplasmic reticulum cisternae) was carried out on $10 \mathrm{EM}$ pictures taken from each EMB sample, showing the complete area of at least one cell (x1500-x3000 magnification). The area of the entire cells and that of each subcellular component (calculated as percentage of total mean cell area) was measured by a computerassisted image analyzer equipped with KS-300 software (Carl Zeiss Co, Germany, rel.1995). The mean total cell area was calculated on the total area of 16 myocardiocytes.

\subsubsection{Assessment of myocardial aldosterone receptors and aquaporin channels}

2.2.3.1. Immunohistochemistry. The expression of aldosterone receptors and aquaporins was evaluated by immunoperoxidase using mineralocorticoid receptor monoclonal antibody (1:100, Enzo Life Sciences, Inc. 10 Executive Blvd Farmingdale, NY 11735 U.S.A.) and Aquaporin 1 and 4 (AQP1, AQP4) mouse monoclonal antibody (1:80 and 1:10 o.n. Santa Cruz Biotechnology, Inc. 10410 Finnell Street Dallas, Texas 75220 U.S.A.) as primary antibodies. Intensity of immunostaining was semi quantitatively evaluated as absent (grade 0 ), weak (grade 1 ), mild (grade 2 ) moderate (grade 3 ), strong (grade 4). For each patient the grading was calculated on 10 different histological section and the average value was computed.

2.2.3.2. Protein isolation and western blot. Heart tissue samples were treated as described [11]. The expression of myocardial aldosterone receptors and Aquaporin 1 and 4 was visualized by using mineralocorticoid receptor monoclonal antibody (1:500), anti-Aqp1 (1:100) and anti-Aqp4 (1: 200). Anti- $\alpha$-sarcomeric actin (1:500, Sigma-Aldrich), antibody was used for normalization. Signal was visualized using a secondary horseradish peroxidase-labeled goat anti-mouse antibody (goat anti-mouse IgG-HRP 1:5000, Santa Cruz Biotechnology) and enhanced chemiluminescence (ECL Clarity Bio-Rad). The purity as well as equal loading of the fraction was determined by measuring $\beta$-actin protein levels. Digital images of the resulting bands were quantified by the Image Lab software package (Bio-Rad Laboratories, Munchen, Germany) and expressed as arbitrary densitometric units.

\section{Results}

Comparison data among the three groups are reported in Table 1.

\subsection{Cardiac studies}

Coronary arteries were normal in all. Aldosteronism cardiomyopathy was characterized by reduced contractility (Fig. 1 panels A, B) and increased myocardial mass (Fig. 2 panel A). Areas of LGE after gadolinium infusion were detectable in the IV septum and lateral LV free wall suggesting increased myocardial fibrosis (Fig. 2 panel D). Myocardial hypertrophy and fibrosis were more pronounced compared with hypertensive cardiomyopathy and normal plasma aldosterone levels (Fig. 1 Supplementary Material). In one patient, images showed a significant increase in T2 relaxation time calculated with T2 mapping (Fig. 2 panel C: $57 \pm$ $12 \mathrm{~ms}$; normal value: $52 \pm 3 \mathrm{~ms}$ ) which was compatible with a intra myocardial water-content increase and not depicted with conventional T2-weighted STIR (Fig. 2 panel B).

\subsection{Endomyocardial biopsy studies}

In aldosteronism-associated cardiomyopathy (group A) histology showed a volume increase of cardiomyocytes, containing large intracellular vacuoles (Fig. 1 panel C and D, Fig. 2 panel E).
At ultrastructural examination, vacuoles were filled of an electronclear homogeneous content, suggesting ion and water accumulation (Fig. 1 panel E, Fig. 2 panel F-H). Vacuoles were diffusely present inside the cytoplasm and were enclosed by a single membrane, closely associated with sarcoplasmic reticulum and Golgi apparatus (Fig. 1 panel $\mathrm{G}-\mathrm{H})$, suggesting that they may originate upon dilatation of cisternae of sarcoplasmic reticulum and Golgi. Also mitochondria and lysosomes appeared electro-clear likely due to a dilution of their matrix upon ion and water increase (swelling). The interstitial space was widened because of both interstitial edema (amorphous electron-clear spaces) and fibrosis (14.9 $\pm 3.8 \%$ of morphometric endomyocardial samples area, which was 2.5 times higher in group A vs group B and 10 times vs group C (Fig. 1 panel $F$ ). Taken together, ultrastructural changes indicate that ion and water homeostasis is impaired causing an abnormal water compartmentation (vacuolar degeneration and interstitial edema). Finally, several areas of myofibrillolysis and numerous autophagosomes were also sometimes observed, as result of myocardiocyte damage.

Immunohistochemistry for aldosterone receptors showed a lower grading in A vs B and C (Fig. 2 supplementary material panels A-C) because of aldosterone competitive binding [12]. Aquaporin 1 was highly expressed in the cytoplasm of cardiomyocytes, smooth muscle cells and endothelial cells, while it was weak and localized only in endothelial cells in group B and C (Fig. 2 supplementary material panel D-F),

Western blot for mineralocorticoid receptor showed in group A 2.8 fold increase in protein expression compared with group $B$ and 3 fold increase compared with $\mathrm{C}$. Western blot for aquaporin 1 showed in group $\mathrm{A}$ 2.8 fold increase compared with B and 3 fold compared with C. Myocardial aquaporin 4 was undetectable at immunohistochemistry and protein analysis in all groups. The overexpression and the intracellular localization of Aquaporin 1 suggested that this channel might be responsible for the water movement among the intracellular compartments.

\subsection{Myocardial changes induced by hyperaldosteronism are reversible upon adenomectomy and aldosterone normalization}

The 69-year old patient with aldosteronism cardiomyopathy who underwent removal of adrenal adenoma and aldosterone normalization showed at 12 months CMR follow-up a reduction of myocardial mass (avg LV thickness from 13.6 to $11.4 \mathrm{~mm}$ ) with recovery of contractility (LVEF from 38 to 55\%) while LGE positive areas remained unchanged (Fig. 3). T1 and T2 mapping were unaffected either at baseline or at recovery suggesting they were unable to relieve the intracellular water accumulation.

At control histology cardiomyocytes appeared smaller (cell diameter at nuclear level of $20 \pm 7 \mu \mathrm{m}$ vs $29.05 \pm 7.41$ baseline value) and devoid of intracellular clear vacuoles, that were no more detectable in the cytoplasm at both optical and ultrastructural examination (Fig. 3). In addition, the other organelles (chiefly mitochondria and lysosomes) recovered their normal volume and the electron density of their matrix. Myofibrillolysis as well as autophagocytosis phenomena were no more visible. Finally, the extracellular spaces appeared reduced, although the fibrosis was unchanged at comparative morphometric analysis.

\section{Discussion}

Aldosterone receptors, besides their major role on kidney, where they regulate electrolyte and water transport in the distal part of the nephron, have been localized in animal [12] and human heart and vessels and fibroblasts [1]. Hyper-stimulation of cardiovascular aldosterone receptors in human is associated with hypokalemic arterial hypertension, myocardial hypertrophy with sarcomere gene overexpression, left ventricular diastolic and systolic dysfunction and fibrosis [2,3]. In addition, an increased rate of cardiovascular events has been reported in patients with primary aldosteronism compared with those patients with essential hypertension of equivalent severity suggesting a direct myocardial damage from aldosterone overproduction [13]. This is 


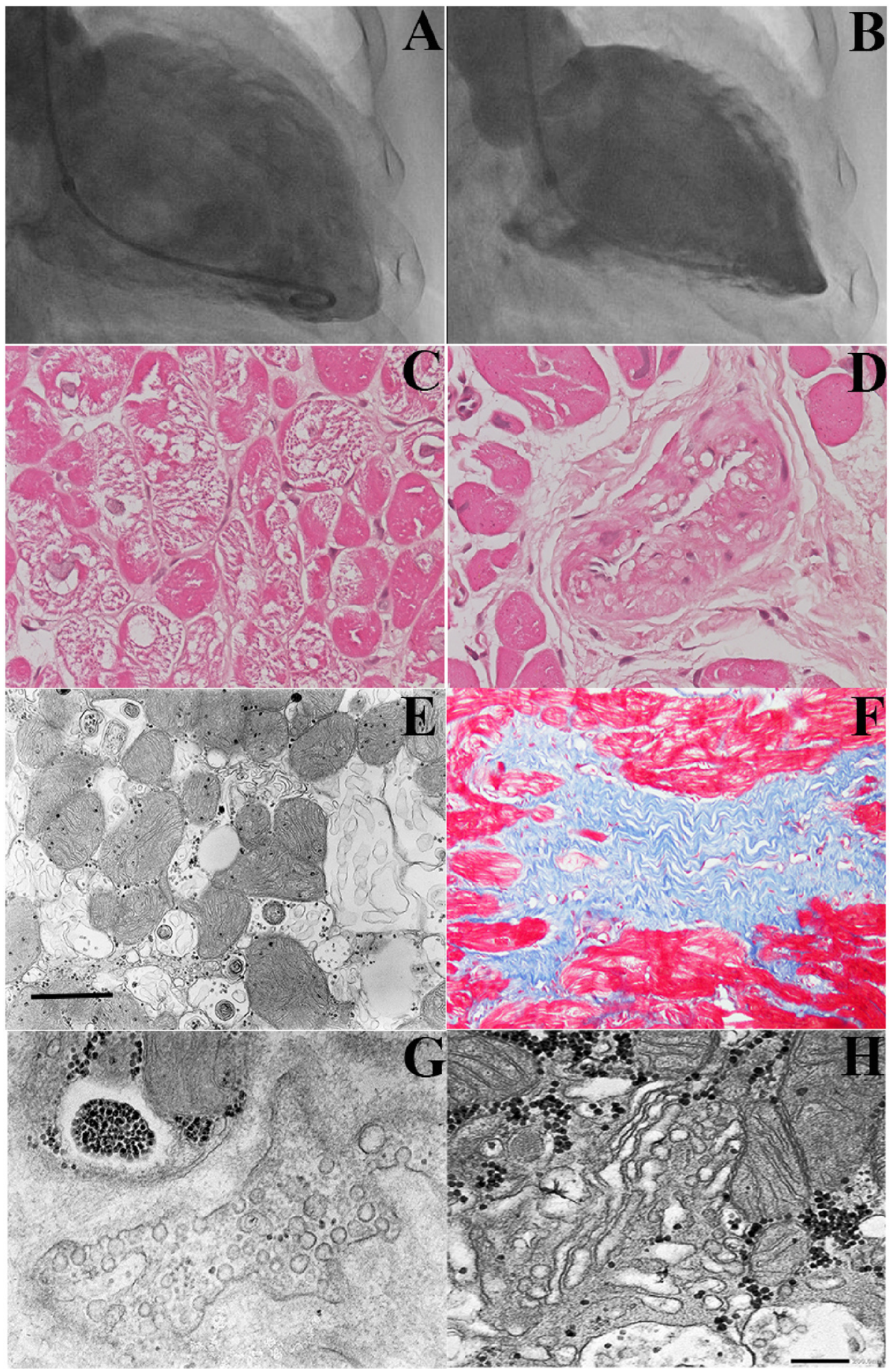

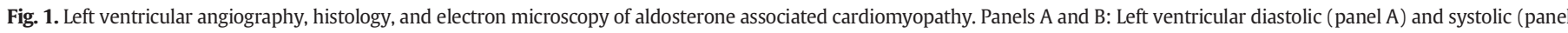

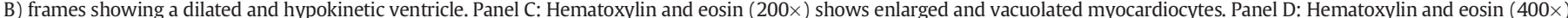

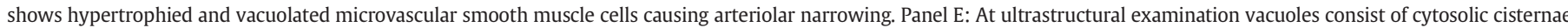

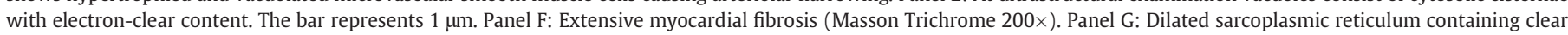
vacuoles. Panel H: Vacuoles affect diffusely Golgi apparatus. The bar represents $1 \mu \mathrm{m}$.

confirmed by the evidence that normotensive patients with very early familial hyperaldosteronism present a thicker left ventricular wall and a reduced diastolic function compared with case-matched normotensive controls [4].

Nevertheless, no detailed pathologic study is available so far in the literature analyzing the morphological changes of cardiomyopathy induced by hyperaldosteronism to be correlated with clinical features. Indeed, as a morphological damage, only myocardial fibrosis is reported in experimental model of hyperaldosteronism [14], and this, if it may account for cardiac diastolic dysfunction, seems unlikely to explain the occurrence of systolic dysfunction as well as the benefits from aldosterone inhibitors reported in patients with heart failure [5-8].

\subsection{Myocardial ultrastructural changes associated with hyperaldosteronism}

Our study, reporting endomyocardial biopsy findings in four patients with hyperaldosteronism-associated cardiomyopathy, shows a 


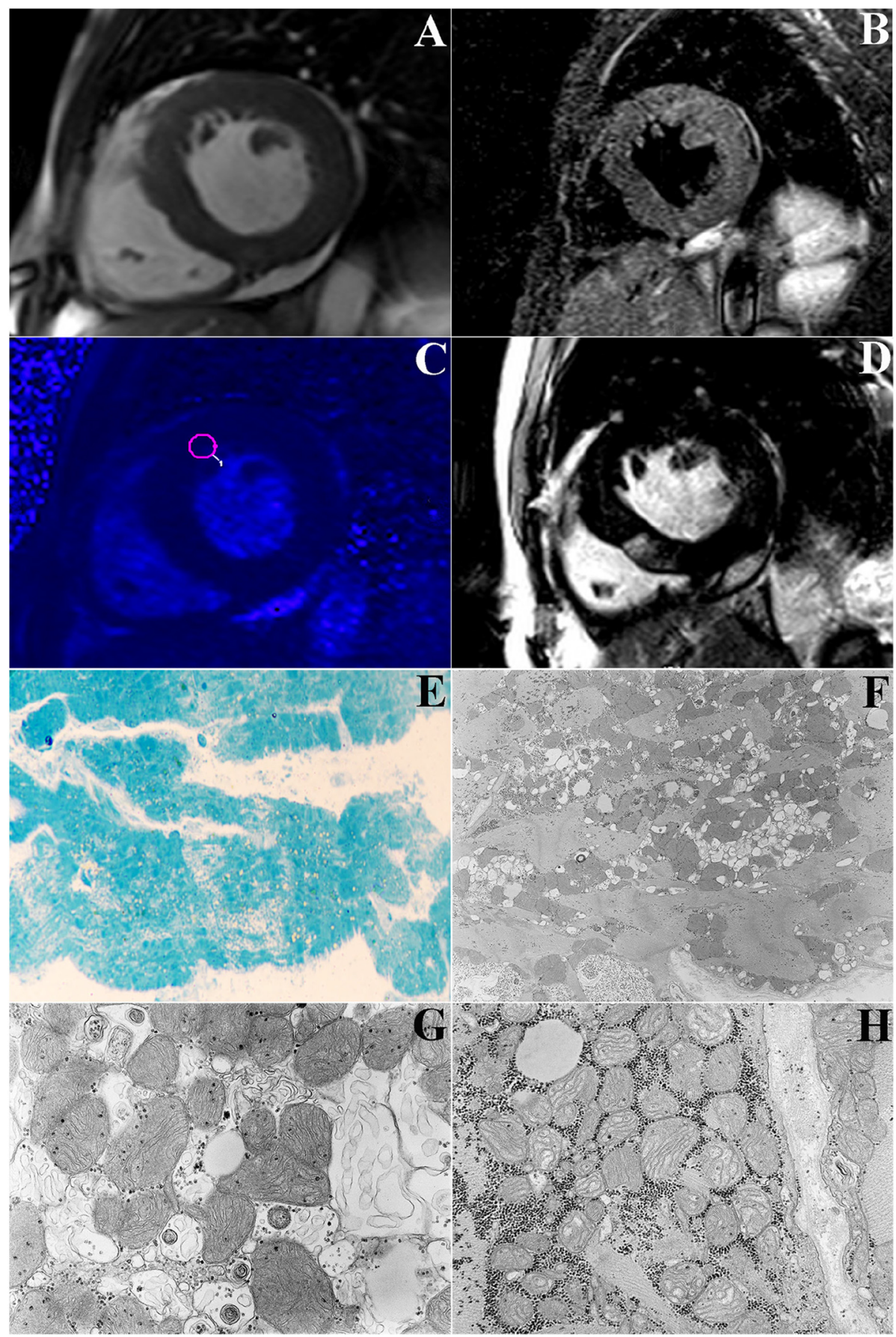

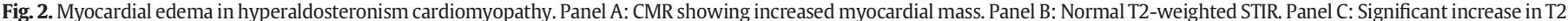

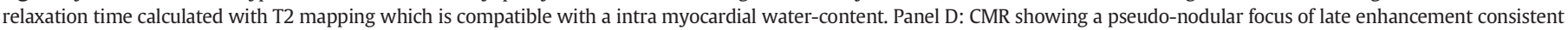

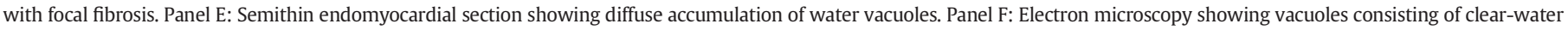
content. Panel G: Ultrastructural examination showing cytosolic water cisternae. Panel H: Ultrastructural evidence of mitochondrial swelling.

remarkable involvement of cardiomyocytes that appear enlarged (hypertrophic) with a number of other subcellular maladaptive changes. Hypertrophy may result from two concurrent mechanisms: a) retention of water and ions (swelling the cytosol and vacuoles) [15]; b) increase in sarcomere and mitochondrial mass. The first is mediated by nuclear receptor-dependent gene expression (ion channels, transporters and aquaporins) [16], the second has been shown as dependent on G protein coupled receptor kinase 5 (GRK5) which exert its pro-hypertrophic effect through a non-canonical cytosolic signaling of aldosterone receptors [17].
Disruption of ion and water homeostasis, as indicated by serum electrolyte changes (increase of $\mathrm{Na}+$ and decrease $\mathrm{K}+$ ), has been described in detail in kidney as a consequence of abnormal aldosteronedependent expression of ion channels, transporters and aquaporins. Vacuoles form as a consequence of water and ion movements among different subcellular compartments and appear likely derived from dilated cisternae of sarcoplasmic reticulum, Golgi apparatus, lysosomes. Cytosol and mitochondrial matrix are also diluted.

Aldosterone, beside its key-regulator of ion and water homeostasis through nuclear receptor-dependent gene expression, shows non- 
canonical activities of $\mathrm{G}$ protein-coupled receptor kinases recently described as responsible, at least in part, for myocardial pathology associated to the hyperaldosteronism. In fact, cardiac hypertrophy and ven-
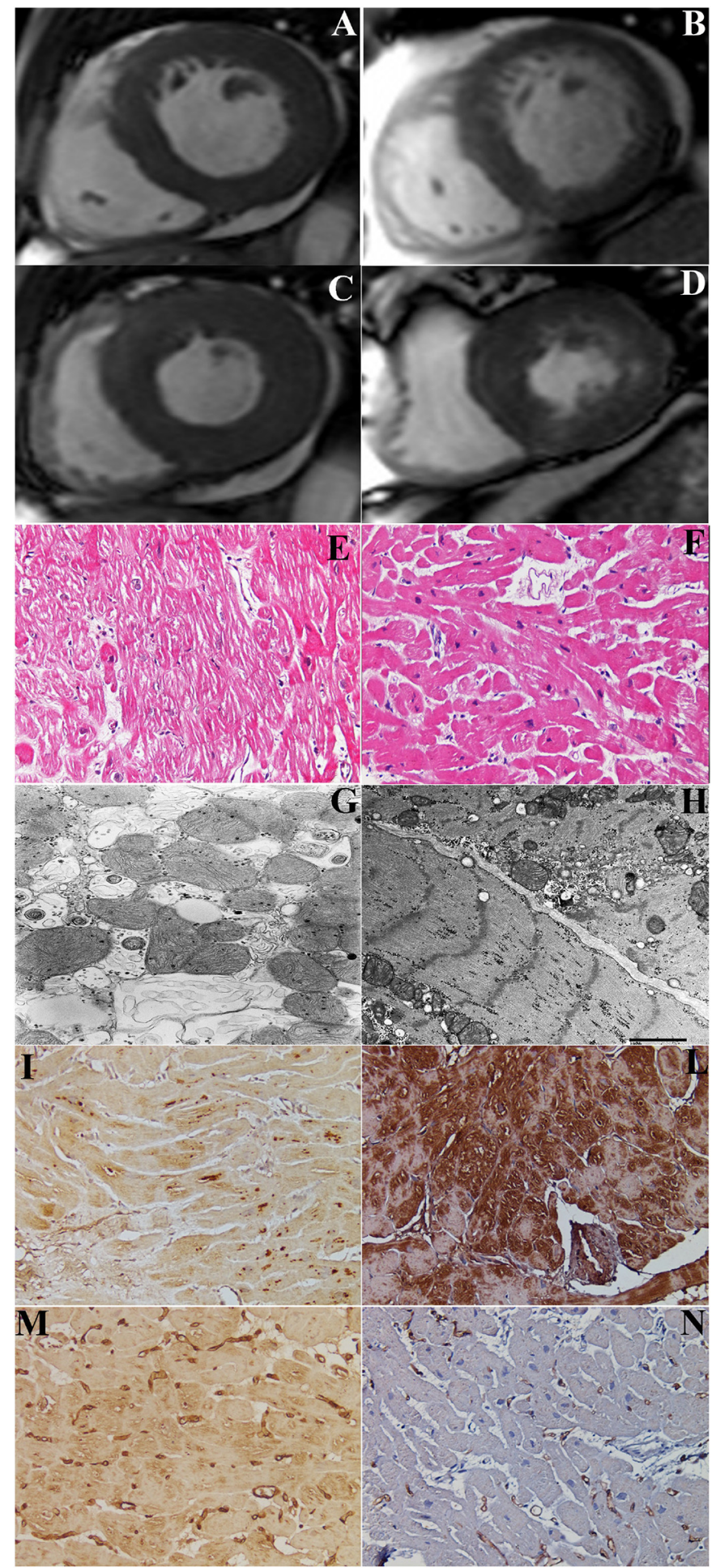

$\mathbf{O}$

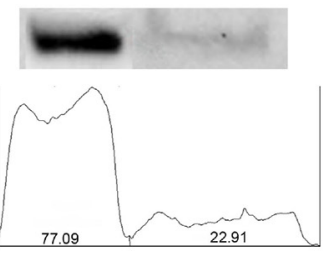

$\mathbf{P}$

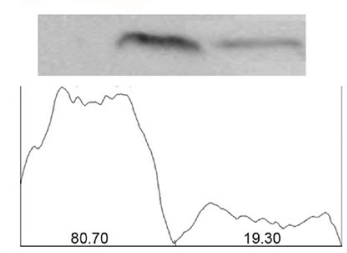

tricular dysfunction may be prevented in an experimental model GRK5 KO mice [17].

This suggests that a crucial consequence of both cell swelling/ vacuolization and maladaptive hypertrophy is a progressive alteration of myofibrillar architecture (disarray), which causes a progressive impairment of cardiac contractility and limitation of cardiomyocyte relaxation. This also may explain diastolic dysfunction since the early phase of aldosteronism, when blood pressure is still normal and a reactive (perivascular and interstitial) myocardial fibrosis is still unlikely.

At histological examination, even intramural vessels appear involved showing hypertrophy and hyperplasia of smooth muscle cells that also, differently from essential hypertension, denotes a clear swelling because of water vacuoles concurring to lumen narrowing.

These findings partly confirm previous in vitro and animal studies [18-20] on aldosterone-mediated remodeling of small resistance arteries and suggest the coexistence of myocardial hypoperfusion in primary aldosteronism cardiomyopathy.

At long term, myocardial fibrosis has been advocated [14-20] as a major pathologic consequence of aldosterone hypersecretion. Indeed, cardiac fibroblasts are known to have high affinity receptors for aldosterone and account for the accumulation of collagen within the interstitium of rat myocardium exposed to increased aldosterone levels. The molecular pathway involved includes a direct aldosterone action as well as an autocrine release of growth factor endotelin-1 [21]. Morphometric quantification of myocardial fibrosis in our four patients with aldosteronism cardiomyopathy reported prominent values (around $14 \%$ ) of fibrosis, 2.5 fold on average higher than those on comparable essential hypertension (6\%).

Intracellular vacuolization and swelling was not found in the myocardial biopsies of patients with essential hypertension, normal aldosterone and comparable compromise of left ventricular function, confirming a specific aldosterone-mediated myocardial toxicity.

Our results, although still incomplete, are in agreement with proposed mechanisms for clinical and subcellular damages observed in the cardiomyopathy of our group of patients. In particular, the increase of aldosterone receptors and aquaporin 1, electrolyte alterations, vacuole formation, mitochondrial and cytosol swelling and hypertrophic sarcomeric disarray all converge in the alteration of contractility/relaxation properties and, at long term, in myocardiocyte damage and myofibrillolysis leading to fibrotic repair and progressive functional deterioration.

\subsection{Effects of adenomectomy and aldosterone normalization on changes associated with hyperaldosteronism}

Structural changes affecting the myocardium after aldosterone normalization have never been provided before. Comparison between baseline and control LV endomyocardial biopsies shows a remarkable myocardial reduction in aldosterone receptors and aquaporin 1 . These biological events have been associated with reduction of cardiomyocyte size, manifesting disappearance of water vacuoles, mitochondrial and cytosolic swelling and reduction of hypertrophic disarray of myofibrillolysis and autophagosomes. These findings suggest that aldosteronism-associated myocardial hypertrophy that may also

Fig. 3. CMR, histology, electron microscopy, immunostaining and western blot quantification of aldosterone and aquaporin 1 receptor in PACM before and after adrenalectomy. Panel A: CMR in diastolic frame showing increase myocardial mass. Panel B: CMR in systolic frame showing reduced left ventricular function. Panel C: Reduced end-diastolic diameter and myocardial mass after adrenalectomy. Panel D: Recovery of left ventricular function but persisting LGE signal with aldosterone normalization. Panel E: Cardiomyocyte hypertrophy and vacuolization reducing (Panel F) after aldosterone normalization. Hematoxylin and eosin (200x). Panel G: Electron microscopy showing water cisternae disappearing (Panel $\mathrm{H}$; the bar represents $1 \mu \mathrm{m}$ ) after adrenalectomy. Panel I: Weak immunostaining for aldosterone receptors that reverts after adrenalectomy (Panel L) (200x). Panel M: Increased immunostaining for aquaporin 1 in PACM that disappears (Panel N) with adrenalectomy $(200 \times)$. Panel O: Western blot of aldosterone receptors showing 2.8 increase that declines after aldosterone normalization. Panel P: Western blot of aquaporin 1 receptors showing 2.8 increase that declines after adrenalectomy. 
precede the development of systemic hypertension, could be mainly due to reversible intracellular water overloading causing myocyte stiffness and degradation of contractile elements followed by enhancement of cell autophagocytosis. Myocardial water accumulation can be appreciated in some PACM patients with pronounced LV wall thickness.

Myocardial fibrosis, appeared more prominent than in primary hypertension, was unchanged at morphometric as well as CMR (LGE) evaluation and suggest that is likely an irreversible phenomenon.

If these data will be confirmed and completed by further studies, they would suggest that even secondary states of aldosteronism as those documented in heart failure may concur to myocardial and vascular water overloading and a maladaptive hypertrophy, which on one side may influence the impairment of cardiovascular system and, on the other, emphasize the possible therapeutic role of aldosterone inhibitors.

\section{Limitation of the study}

Although primary aldosteronism-associated cardiomyopathy is an uncommon disorder and the histological, ultrastructural and molecular findings are uniform in all 4 patients investigated, the small cohort involved makes the study suggestive but not definitive. A wider report is needed for confirmation.

In conclusions PA cardiomyopathy is a reversible entity characterized by an overexpression of myocardial aldosterone receptors and AQP 1 channels with cardiomyocyte and microvascular water accumulation, maladaptive hypertrophy, areas of myofibrillolysis and impairment of myocyte relaxation and contraction.

\section{Funding}

This work was supported by AIFA grant "Multicenter randomized study on the efficacy of immunosuppression in patients with virusnegative inflammatory cardiomyopathy" (FARM12JCXN) and by European Project ERA-CVD “Transnational Research Projects on Cardiovascular Diseases” (JTC 2016 IKDT-IGCM).

\section{Declaration of Competing Interest}

None.

\section{Appendix A. Supplementary data}

Supplementary data to this article can be found online at https://doi. org/10.1016/j.ijcard.2019.06.055.

\section{References}

[1] M. Lombès, N. Alfaidy, E. Eugene, A. Lessana, N. Farman, J.P. Bonvalet, Pre-requisite for cardiac aldosterone action. Mineralocorticoid receptor and 11-betahydroxysteroid dehydrogenase in the human heart, Circulation 92 (1995) 175-182.

[2] J.W. Funder, Aldosterone and mineralocorticoid receptors. Physiology and pathophysiology, Int. J. Mol. Sci. 18 (2017) (pii: E1032).

[3] M. Cesari, C. Letizia, P. Angeli, S. Sciomer, S. Rosi, G.P. Rossi, Cardiac remodeling in patients with primary and secondary aldosteronism. A tissue Doppler study, Circ Cardiovasc Imaging 9 (2016), e004815.

[4] M. Stowasser, J. Sharman, R. Leano, R.D. Gordon, G. Ward, T.H. Marwick, Evidence for abnormal left ventricular structure and function in normotensive individuals with familial hyperaldosteronism type I, J. Clin. Endocrinol. Metab. 90 (2005) 5070-5076.

[5] W.J. Emme, R. Cody, A. Castaigne, A. Perez, J. Palensky, J. Wittes, The effect of spironolactone on morbidity and mortality in patients with severe heart failure, N. Engl. J. Med. 341 (1999) 709-717.

[6] B. Pitt, W. Remme, F. Zannad, et al., A selective aldosterone blocker, in patients with left ventricular dysfunction after myocardial infarction, N. Engl. J. Med. 348 (2003) 1309-1321.

[7] B. Pitt, G. Bakris, L.M. Ruilope, L. Di Carlo, R. Mukherjee, EPHESUS investigators, Serum potassium and clinical outcomes in the Eplerenone Post-Acute Myocardial Infarction and Heart Failure Efficacy and Survival Study (EPHESUS), Circulation 118 (2008) 1643-1650.

[8] F. Zannad, J.J. McMurray, H. Krum, et al., Eplerenone in patients with systolic heart failure and mild symptoms, N. Engl. J. Med. 364 (2011) 11-21.

[9] C.W. Yancy, M. Jessup, B. Bozkurt, et al., 2013 ACCF/AHA guideline for the management of heart failure, Circulation 128 (2013) 1-375.

[10] C.W. Yancy, M. Jessup, B. Bozkurt, et al., 2016 ACC/AHA/HFSA update on new pharmacological therapy for heart failure: an update of the 2013 ACCF/AHA guideline for the management of heart failure, Circulation 134 (2016) 1-18.

[11] C. Chimenti, R. Verardo, F. Scopelliti, et al, Myocardial expression of Toll-like receptor 4 predicts the response to immunosuppressive therapy in patients with virusnegative chronic inflammatory cardiomyopathy, Eur. J. Heart Fail. 19 (2017) 915-925.

[12] M. Lombès, M.E. Oblin, E.E. Baulieu, N. Farman, J.P. Bonvalet, Immunohistochemical and biochemical evidence for a cardiovascular mineralocorticoid receptor, Circ. Res. 71 (1992) 503-510.

[13] P. Milliez, X. Girerd, P.F. Plouin, J. Blacher, M.E. Safar, J.J. Mourad, Evidence for an increased rate of cardiovascular events in patients with primary aldosteronism, J. Am. Coll. Cardiol. 45 (2005) 1243-1248

[14] K.T. Weber, C.G. Brilla, S.E. Campbell, E. Guarda, G. Zhou, K. Sriram, Myocardial fibrosis: role of angiotensin II and aldosterone, Basic Res. Cardiol. 88 (Suppl. 1) (1993) 107-124.

[15] A. Rutkovskiy, G. Valen, J. Vaage, Cardiac aquaporins, Basic Res. Cardiol. 108 (2013) 393-396.

[16] M.A. Russo, E. Morgante, A. Russo, G.D. van Rossum, M. Tafani, Ouabain-induced cytoplasmic vesicles and their role in cell volume maintenance, Biomed. Res. Int. 2015 (2015), 487256.

[17] A. Cannavo, D. Liccardo, A. Eguchi, et al., Myocardial pathology induced by aldosterone is dependent on non-canonical activities of $G$ protein-coupled receptor kinases, Nat. Commun. 7 (2016), 10877.

[18] E.L. Schiffrin, The many targets of aldosterone, Hypertension 43 (2004) 938-940.

[19] D. Rizzoni, E. Porteri, M. Castellano, et al., Vascular hypertrophy and remodeling in secondary hypertension, Hypertension 28 (1996) 785-790.

[20] C.G. Brilla, Aldosterone and myocardial fibrosis in heart failure, Herz (3) (2000) 299-306.

[21] L.C. Katwa, K.T. Weber, Aldosterone-induced expression of ET1, ET3 and ETB receptors by cultured adult rat cardiac fibroblasts, Circulation 94 (1996) I-146. 